Published in final edited form as:

Am J Gastroenterol. 2017 June ; 112(6): 940-947. doi:10.1038/ajg.2017.6.

\title{
Successful Resolution of Recurrent Clostridium difficile Infection using Freeze-Dried, Encapsulated Fecal Microbiota; Pragmatic Cohort Study
}

\author{
Christopher Staley, $\mathrm{PhD}^{1,4}$, Matthew J. Hamilton, $\mathrm{PhD}^{1,4}$, Byron P. Vaughn, MD², Carolyn T. \\ Graiziger, BS ${ }^{2}$, Krista M. Newman, MD $^{2}$, Amanda J. Kabage, MS $^{2}$, Michael J. Sadowsky, \\ PhD ${ }^{1,5}$, and Alexander Khoruts, MD ${ }^{1,2,3,5}$ \\ ${ }^{1}$ BioTechnology Institute, University of Minnesota, St Paul, MN, USA \\ 2Division of Gastroenterology, Department of Medicine, University of Minnesota, Minneapolis, \\ MN, USA \\ ${ }^{3}$ Center for Immunology, University of Minnesota, MN, USA
}

\begin{abstract}
OBJECTIVES-Fecal microbiota transplantation (FMT) is increasingly being used for treatment of recurrent Clostridium difficile infection (R-CDI) that cannot be cured with antibiotics alone. In addition, FMT is being investigated for a variety of indications where restoration or restructuring of the gut microbial community is hypothesized to be beneficial. We sought to develop a stable, freeze-dried encapsulated preparation of standardized fecal microbiota that can be used for FMT with ease and convenience in clinical practice and research.
\end{abstract}

METHODS-We systematically developed a lyophilization protocol that preserved the viability of bacteria across the taxonomic spectrum found in fecal microbiota and yielded physicochemical

Correspondence: Alexander Khoruts, MD, University of Minnesota, Professor of Medicine, 2101 6th Street S.E.; Room 3-184, Wallin Biomedical Sciences Building, Minneapolis, MN 55414, USA. khoru001@umn.edu or Michael J. Sadowsky, PhD, McKnight University Professor, University of Minnesota, BioTechnology Institute, 1479 Gortner Avenue, 140 Gortner Labs, St. Paul, MN 55108, USA.sadowsky@umnedu.

${ }^{4}$ Shares first authorship.

${ }^{5}$ Shares senior authorship.

\section{CONFLICT OF INTEREST}

Guarantor of the article: Alexander Khoruts, MD.

Specific author contributions: Christopher Staley: analysis and interpretation of data; critical revision of the manuscript for intellectual content; statistical analysis. Matthew J. Hamilton: acquisition of data; analysis and interpretation of data; critical revision of the manuscript for intellectual content. Byron P. Vaughn: acquisition of data; critical revision of the manuscript for intellectual content. Carolyn T. Graiziger: acquisition of data; technical support. Krista M. Newman: acquisition of data. Amanda J. Kabage: administrative and technical support. Michael J. Sadowsky: study concept and design; analysis and interpretation of data; critical revision of the manuscript for intellectual content; obtained funding; study supervision. Alexander Khoruts: study concept and design; acquisition of data; analysis and interpretation of data; drafting the manuscript; obtained funding; study supervision.

Financial support: The study was supported by grants from the NIH 1R21-AI114722-01 (AK, MJS) to analyze the bacterial composition of the fecal samples, CIPAC Limited (AK, MJS) to test various parameters for optimization of microbiota preparation. Additional support for capsule preparation and collection biological specimens was provided by the philanthropic support of Achieving Cures Together, Ms. Emily Haller, and the Hubbard Foundation. None of the sponsors had any roles (other than funding) in the study design, collection, analysis, interpretation of data, or writing of the manuscript.

Potential competing interests: AK and MJS received research grant support from CIPAC Limited. MJH, AJK, and MJS provided consulting services to CIPAC Limited and Crestovo LLC. AK serves on the advisory board for Merck. BPV receives research salary support from Roche and speaking consulting fees from Janssen and Abbvie. The remaining authors declare no conflict of interest. SUPPLEMENTARY MATERIAL is linked to the online version of the paper at http://www.nature.com/ajg 
properties that enabled consistent encapsulation. We also treated a cohort of R-CDI patients with a range of doses of encapsulated microbiota and analyzed the associated changes in the fecal microbiome of the recipients.

RESULTS-The optimized lyophilized preparation satisfied all our preset goals for physicochemical properties, encapsulation ease, stability at different temperatures, and microbiota viability in vitro and in vivo (germ-free mice). The capsule treatment was administered to 49 patients. Overall, $43 / 49$ (88\%) of patients achieved a clinical success, defined as no recurrence of CDI over 2 months. Analysis of the fecal microbiome demonstrated near normalization of the fecal microbial community by 1 month following FMT treatment. The simplest protocol using the lowest dose (2.1-2.5 $\times 10^{11}$ bacteria in 2-3 capsules) without any colon purgative performed equally well in terms of clinical outcomes and microbiota engraftment.

CONCLUSIONS-A single administration of encapsulated, freeze-dried fecal microbiota from a healthy donor was highly successful in treating antibiotic-refractory R-CDI syndrome.

\section{INTRODUCTION}

Widespread use of antimicrobial drugs over many decades in medicine and agriculture has resulted in the emergence of increasing numbers of antibiotic-resistant pathogens, which constitute one of the most urgent growing threats in modern healthcare $(1,2)$. In addition, antibiotics increase vulnerability to infections by lowering colonization resistance that is normally provided by the host's own microbiota (3). Therefore, there is a need for more targeted treatments against pathogens that can spare the host microbiota, and/or restorative treatments that can recover the normal composition of gut microbiota.

The syndrome of recurrent Clostridium difficile infection (R-CDI) is a common clinical challenge that captures the essential pitfalls of reliance on broad-spectrum antibiotics for treatment (4). The standard antibiotics for this infection, e.g., metronidazole and vancomycin, suppress gut microbiota leading to a disruption of normal microbial community structure, dysbiosis, and a decrease in overall microbial diversity. CDI reoccurs after cessation of antibiotic therapy, when the loss of protective microbiota allows $C$. difficile spore germination, expansion of vegetative forms of $C$. difficile bacteria and production of endotoxins. Restoration of the gut microbial community structure and function via fecal microbiota transplantation (FMT) - transplantation of the gut microbial community from healthy donor stool—has emerged as a highly effective treatment of refractory R-CDI $(5,6)$.

FMT has developed rapidly over the last several years from a crude procedure involving different routes of administration of raw stool from patient-identified donors to the use of purified and cryopreserved standardized preparations of fecal microbiota from highly selected donors (7). Although colonoscopic administration of FMT has the advantage of a direct application providing potentially clinical relevant diagnostic information (8), an oral preparation is preferable for many patients due to its greater ease of administration (9). Encapsulated, frozen microbiota can be delivered orally and results in successful treatment of R-CDI (10); however, the practicality of this preparation is somewhat limited by uniformity, storage, and shelf-life issues. In addition, little is known about microbiome changes that follow capsule FMT. 
We sought to develop the next-generation form of encapsulated FMT using a freeze-dried preparation of microbiota that could tolerate a range of temperatures to allow ease of handling and satisfy the following conditions: (i) the freeze-drying procedure should preserve viability and diversity of the entire taxonomic spectrum of microbiota, (ii) the resulting material should have physicochemical properties that enable standardized encapsulation, (iii) the encapsulation procedure should not compromise the viability of microbiota, and (iv) the microbiota should engraft into the colon and successfully treat RCDI. Here we describe development of such a preparation and the results of its use in clinical practice.

\section{METHODS}

\section{Freeze-dried fecal microbiota}

Fecal bacteria were prepared using the standard methods as previously described (7), except that glycerol was substituted with one of the following cryoprotectants (all chemicals were USP grade or better and prepared in PBS, pH 7.0): $5 \%$ sucrose only; $10 \%$ sucrose only; $10 \%$ skim milk only; $5 \%$ trehalose only; $10 \%$ trehalose only; $10 \%$ trehalose plus $2.5 \%$ sucrose; $5 \%$ trehalose plus $2.5 \%$ sucrose; $5 \%$ mannitol only; or $10 \%$ mannitol only. The lyophilizer (LyoStar II, Stone Ridge, NY) used a shelf temperature of $-20^{\circ} \mathrm{C}$ for $36 \mathrm{~h}$ followed by $6 \mathrm{~h}$ at $+30^{\circ} \mathrm{C}$. All steps were done under $100 \mathrm{mT}$ vacuum or less, and the final product was held at $+20^{\circ} \mathrm{C}$ until used. The total dose was $2.5 \times 10^{12}$ cells. Cell numbers were determined by using the Bacteria Counting Kit (ThermoFisher, Waltham, MA), a Zeiss epifluorescense microscope equipped with a 470-490 $\mathrm{nm}$ excitation filter and $500 \mathrm{~nm}$ cut-on long pass emission filter, and a Petroff Hauser counting chamber. Membrane integrity procedures were also used to directly enumerate microbes present in a diluted sample and to calculate the percentage of microbes with intact membranes present in the intermediate fecal slurry. This was done using a BacLight live/dead membrane integrity assay (ThermoFisher, Waltham, MA) and the microscope and counting chamber described above.

Mice

Germ-free mice were bred and maintained in the germ free facility at the Mayo Clinic (Rochester, MN, USA). Animals were administered microbiota or PBS via oral gavage needle, $100 \mu \mathrm{l}$ per dose. Microbiota preparations included frozen/thawed liquid with 10\% glycerol, as described previously or rehydrated microbiota freezedried in 5\% trehalose. The dosage administered to each mouse, of either frozen or freezedried material, was $10^{10}$ cells. Fecal pellets were collected before gavage, as well as at 3, 7, 14, and 21 days following gavage.

\section{Preparation of FMT capsules}

Double-encapsulated capsules were prepared by using a filled size 0 capsule packaged inside a size 00 capsule. Hypromellose capsules were DRcaps from Capsugel (Morristown, NJ). Capsules were manually filled using a 24-hole filler (Capsule Machine, Capsule Connection, Prescott, AZ) to a final concentration of $\sim 1 \times 10^{11}$ cells/capsule. The capsules were stored at $-80{ }^{\circ} \mathrm{C}$ (a convenient dry storage option) in $50 \mathrm{ml}$ conical tubes until needed. Once removed 
from the freezer, a $1 \mathrm{~g}$ silica gel canister (Dry Pak Industries, Encino, CA) was added to the container.

\section{Patient population}

All patients in this cohort, satisfied formal inclusion and exclusion criteria, described previously for the University of Minnesota clinical FMT program (8). Briefly, these inclusion criteria were: (i) informed consent; (ii) documentation of at least two spontaneous recurrences of CDI following the initial episode of the infection; (iii) failure of at least one extended antibiotic regimen ( $\geq$ weeks) to prevent spontaneous recurrence of the infection; (iv) documentation of CDI by stool testing within 3 months of FMT. If the patients qualified for FMT, they were always given the option of microbiota delivery via a colonoscopic route of administration during the consent process. Exclusion criteria for all FMT patients included: (i) anticipation of non-CDI antibiotic treatment within 3 months of FMT; (ii) life expectancy of less than 2 years if the patient is able to tolerate suppressive therapy with vancomycin, $125 \mathrm{mg}$ daily, or rifaximin for patients with liver disease and hepatic encephalopathy. In addition, exclusion criteria for FMT with an encapsulated oral preparation of FMT in this study included: (i) dysphagia, (ii) clinical indications for a diagnostic colonoscopy, including anticipated screening or surveillance colonoscopy within 1 year, hematochezia, iron deficiency anemia, poor symptomatic responsiveness to antibiotic treatment of CDI, past history of unexplained diarrheal symptoms, (iii) any immunosuppressive therapy or presence of known immune deficiency (e.g., IgA deficiency), and (iv) known inflammatory bowel disease, given likely immunosuppression, importance of mucosal examination at the time of FMT, and uncertainty about location of capsule release, e.g., stricture. Patients excluded specifically from capsule FMT were offered colonoscopic FMT as an option if the general FMT inclusion/exclusion criteria were satisfied.

Some patients included in this cohort were recipients of previous FMT, administered via colonoscopy. In our program, patients suffering a spontaneous recurrence of CDI, i.e., recurrence of the infection without a new antibiotic provocation, are offered another round of FMT. If the patients suffer a recurrence following a new antibiotic provocation, they are offered one round of anti-CDI antibiotic (preferably fidaxomicin) and FMT is offered only following a spontaneous recurrence after this antibiotic treatment attempt.

\section{FMT protocol}

Donor material used in preparation of FMT capsules was obtained from standard donors as previously described (University of Minnesota IRB donor protocol 1303M29782) (7). Two male donors provided material for capsule FMT preparations. A research assistant delivered the FMT capsules to patient homes and reinforced instructions for the FMT protocol. Patients were told they could keep the capsules in the refrigerator for 2 days. The patients were allowed only water for $2 \mathrm{~h}$ before taking the capsules, and only water was allowed for 2 $\mathrm{h}$ after taking the capsules during which time the patients had to remain upright. The protocol evolved in the course of clinical experience as described further in the results section. Variables included: (i) administration of a colon purgative before capsule FMT (discontinued after the first four patients; (ii) acid suppressive medications; (iii) the dose of 
fecal microbiota, which was decreased in the course of the study due to limited quantity of prepared material.

\section{Clinical study design}

This is a single center (University of Minnesota) pragmatic study describing the entire clinical experience with capsule FMT from June 2014 to March 2016. Failure of FMT was defined as spontaneous relapse of diarrheal symptoms, and positive stool testing for $C$. difficile toxin B by PCR, within 2 months of administration. Diarrhea was defined as $\geq 3$ loose/watery stools per day for $\geq 2$ consecutive days or a significant change in bowel habits toward diarrhea combined with clinical suspicion for CDI recurrence, e.g., abdominal cramps, fatigue. $C$. difficile toxin B was measured in all patients who noted or complained of loose stools, regardless of frequency. Patients were visited at their home by the study research staff before capsule FMT, at weeks 2 and 4, and longer-term time points at 3 and 612 months post-FMT. Patients were able and encouraged to promptly communicate any concerns at all times to the research and clinic staff, as well the principal investigator. All patients were seen in clinic after 2 months for a follow-up clinic visit following capsule FMT and were instructed to remain in contact with the clinic indefinitely with any new questions or concerns and any new prescriptions for antibiotics by other providers. The study was approved by the University of Minnesota Institutional Review Board (IRB Protocol 0901M56962). The protocol changes in the course of this study and ethics of FMT are further discussed in the Supplementary Material online.

\section{Fecal sample collection}

Fecal samples were collected by patients into sterile containers within a week before FMT, on post-FMT days $3,7,14$, and post-FMT months 1,3 , and 6-12. The samples were kept frozen at $-20{ }^{\circ} \mathrm{C}$ until pick-up by the research staff and transported on ice into the laboratory.

\section{Analysis of fecal microbiota}

DNA from single mouse fecal pellets or 250-500 $\mathrm{mg}$ of human fecal material was extracted using the PowerSoil DNA Isolation Kit (MoBio Laboratories, Inc., Carlsbad, CA, USA) without deviation from the manufacturer's instructions. The microbiome was characterized from patients for whom capsule FMT represented their first intervention (i.e., no prior colonoscopic FMT) and those that experienced any recurrence of infection, regardless of prior intervention. The V5+V6 hypervariable regions of the $16 \mathrm{~S}$ rRNA gene were amplified at the University of Minnesota Genomics Center (UMGC, Minneapolis, MN, USA) using the BSF784/1064R primer set $(11,12)$. Amplicons were gel purified and purified amplicons were pooled in equal amounts for sequencing. Paired-end sequencing was performed by UMGC at a read length of $300 \mathrm{nt}$ using the Illumina MiSeq platform (Illumina, Inc., San Diego, CA, USA). Sequencing data were recovered as fastq files and are deposited in the Sequence Read Archive of the National Center for Biotechnology Information under BioProject accession numbers SRP071210 and SRP064361, for germ-free mice and donor/ patient samples, respectively. 
Sequence processing and analysis, unless otherwise noted, was performed using mothur ver. 1.34.0 (refs. 13,14). Fastq files for both forward and reverse reads were trimmed to $150 \mathrm{nt}$ and paired-end joined using fastq-join soft ware (15). Sequences were quality trimmed at an average quality score of 35 over a window of $50 \mathrm{nt}$. Any sequence with homopolymers $>8$ nt, an ambiguous base, or $>2$ mismatches from primer sequences was excluded. High-quality sequences were aligned against the SILVA database ver. 119 (ref. 16) and subjected to a $2 \%$ pre-clustering step (17). Chimeras were identified and removed using UCHIME software (18). For comparison between samples and data sets, the number of reads per sample was rarefied to 50,456 reads for germ-free mice and 11,500 reads for patient comparisons (19). Operational taxonomic units were assigned at a $97 \%$ identity level using the furthestneighbor algorithm and taxonomic assignments were made against the RDP14 database (20). Engraftment of the donor microbial community was determined as a percentage of recipient communities that could be attributed to donor samples using the default parameters of the SourceTracker software (21).

\section{Statistical analyses}

Differences in alpha diversity, relative abundances of phyla, and donor similarity were evaluated by ANOVA using XLSTAT version 2015.1.01 (Addinsoft, Belmont, MA, USA). Ordination by principal coordinate analysis, evaluation of the significance of grouping, and beta diversity statistics were performed using mothur. Analysis of molecular variance was used to determine if grouping was significant (22), and analysis of similarity was used to evaluate differences in beta diversity (23).

\section{RESULTS}

\section{Optimization and pre-clinical testing of freeze-dried microbiota}

Lyophilization of fecal microbiota preparations is a key step towards generation of a convenient oral dosing form for use in FMT. The use of cryoprotectants in lyophilization is required to prevent the formation of cell wall- and membrane-damaging ice crystals during the freezing and desiccation process. Glycerol, which is typically used as a cryoprotectant in preparation of frozen liquid suspensions of microbiota $(7,24)$, is not useful in lyophilization because the viscosity of the resulting end product is not amenable to encapsulation. Certain alternatives, such as milk products or bovine serum, are not suitable for pharmaceutical use due to potential for allergies and issues with batch variation. We tested a number of potential cryoprotectants and found that mannitol and trehalose (at 5\% and 10\% concentrations) yielded superior preparations that could be ground into a fine powder and easily packaged into capsules. However, viability of bacteria with trehalose was superior to that found with mannitol, as measured by a membrane integrity assay which gives the percentage of the total cells with intact membranes (Supplementary Table 1 online). In addition, the membrane integrity assay showed the viability of the fresh material was nearly indistinguishable compared to lyophilized fecal microbiota (60\% and 56\% intact cells, respectively). Consequently, $5 \%$ trehalose was chosen as our standard cryoprotectant in the rest of the study. 
Since flexibility in storage and handling conditions is an important issue in clinical care delivery, we tested the stability of freeze-dried microbiota at different temperatures. Membrane integrity of freeze-dried microbiota remained intact after $96 \mathrm{~h}$ of storage at room temperature, 4 and $-20{ }^{\circ} \mathrm{C}$ (Supplementary Table 2). The prepared capsules were stored long-term at $-80^{\circ} \mathrm{C}$, chosen as the most convenient dry environment available to us (Supplementary Table 3).

Finally, in order to ensure that the freeze-drying protocol preserved the viability of bacteria in the preparation across the taxonomic spectrum, we measured the engraftment of the preparations in germ-free mice. Comparison was made to frozen/thawed liquid preparation with glycerol, which had previously been demonstrated to retain full viability following FMT in patients (25). Prompt and stable engraftment of all bacterial phyla was evident for both frozen and freeze-dried treatment groups, as discussed in detail in Supplementary Results, (Supplementary Figures 1 and 2).

\section{Clinical performance of capsule FMT}

The basic characteristics of the patients in this clinical cohort were comparable to patients without underlying inflammatory bowel disease offered FMT in our program (Table 1) (8). these patients had an average of 4-5 episodes of CDI since the initial infection and failed all reasonable attempts at breaking the cycle of R-CDI by using antibiotics alone, including an extended course of tapered vancomycin, per inclusion criteria.

The clinical capsule FMT protocol evolved over the course of the program experience. The dose of microbiota was defined by the number of bacteria. The initial protocol used $\sim 2.5 \times 10^{12}$ bacteria contained in 24-27 capsules and included a colon purgative before administration to wash out residual antibiotic. However, given the limited number of FMT capsules that were produced during the development phase, we had to decrease the dose in later stages of this experience. The final protocol used $\sim 2.1-2.5 \times 10^{11}$ bacteria contained in $2-3$ capsules, taken without a preceding purgative as a single dose. The protocol changes and the consent process are described in the Supplementary Material.

The performance of capsule FMT along with different protocol variables are shown in Table 2. The success rate in preventing CDI recurrence over 2 months of follow-up was $87.8 \%$ (43/49 patients) in the entire cohort. the success rate among all patients for whom the capsule treatment was their first FMT was 89.7\% (35/39 patients). One of these patients received broad-spectrum antibiotics during a hospitalization for a complex urinary infection (in the setting of urinary stents) within a day following her capsule FMT and promptly suffered a recurrence of CDI. The success rate in breaking the cycle of CDI recurrence for the lowest dosage of microbiota was $93.3 \%$ (28/30 patients) and $96.2 \%(25 / 26)$ for patients for whom the capsule treatment was their first FMT. Two patients out of the entire cohort suffered a spontaneous recurrence (i.e., no new antibiotic provocation) of CDI after the 2month end point. No serious adverse events (death, hospitalization, emergency room or physician visit for a new problem requiring intervention) occurred in this cohort within 2 months following FMT with the exception of the mentioned patient with the urinary infection. The patients reported no difficulty or esthetic concerns taking the capsules at any 
dose. Approximately a third of patients reported some bowel movement irregularity, bloating, and flatulence in the initial weeks following capsule FMT.

\section{Changes in the fecal microbiome following FMT}

Given the inherent microbiome complexity expected in patients with history of prior FMT, we limited the characterization of the fecal microbiome to samples obtained from patients for whom the capsule treatment was their first FMT. All samples before FMT showed markedly lower microbial diversity compared to the donor microbiota (see Supplementary Table 4). Similar to previous investigations of patients undergoing FMT for R-CDI in our own program and others $(6,25-30)$, these pre-FMT samples demonstrated markedly increased relative abundance of Proteobacteria $(40.7 \pm 4.3 \%$ vs. $2.0 \pm 0.5 \%, P<0.001)$ and reductions in the relative abundances of Firmicutes $(34.4 \pm 3.8 \%$ vs. $55.4 \pm 3.3 \%, P=0.443)$ and Bacteroidetes $(8.5 \pm 2.7 \%$ vs. $39.7 \pm 3.4 \%, P<0.001)$ compared to the donor samples. Capsule FMT was associated with increased microbial diversity, contraction of the relative abundance of Proteobacteria, and an increase in the relative abundances of Firmicutes and Bacteroidetes, discussed in detail in Supplementary Results. Interestingly, the kinetics of these changes were not equivalent among different phyla (Figure 1). In particular, the recovery of Bacteroidetes was somewhat delayed relative to what was seen with colonoscopically administered FMT (25), and its relative abundance did not stabilize until after 1 month following capsule FMT.

In order to assess the contribution of donor microbiota engraftment to the changes in the FMT-associated microbial community structure, we employed SourceTracker soft ware (see Supplementary Materials) (21). Before FMT this analysis attributed a relatively low fraction $(22.6 \pm 4.4 \%)$ of sequence reads in patient samples to the donors. Donor similarity increased at all time points after capsule FMT and was greatest at least 1 month following treatment. Analysis of fecal samples from patients that received high dose capsule FMT (1.25$2.5 \times 10^{12}$ bacteria) vs. low dose $\left(2.1-2.5 \times 10^{11}\right.$ bacteria) did not show any significant dosedependent differences in microbial diversity changes or kinetics of engraftment, further discussed in Supplementary Results. Similarly, the microbiome analysis did not find any deleterious effect of proton pump inhibitors on capsule FMT. Finally, we analyzed the fecal samples from all patients who suffered a recurrence of CDI following a single treatment with capsule FMT, discussed further in Supplementary Results. Following FMT, the microbiomes of these patients generally became taxonomically more similar to donors, except the one who received antibiotics shortly following the treatment. However, the small number of CDI recurrences precluded elucidation of a clear pattern predictive of FMT failure.

\section{DISCUSSION}

Over the last 5 years FMT has rapidly progressed from a rare procedure on the fringes of medicine to a recommended option listed in the mainstream clinical guidelines in management of R-CDI that cannot be managed with standard antibiotics alone (31). To a large degree, this rapid adoption of FMT has been enabled by use of pre-made, cryopreserved microbiota. The frozen/thawed liquid preparations have been found to be 
clinically equivalent to freshly prepared microbiota in treatment of R-CDI $(7,32,33)$ and indistinguishable in the kinetics of donor microbiota engraftment following administration via colonoscopy, enema, or nasogastric tube (25). A similar frozen, encapsulated preparation has also been successful when administered orally $(10,34,35)$. However, the practicality of the frozen, liquid capsules is somewhat limited by issues pertaining to aesthetics, storage, transport, and handling.

Here we described a freeze-dried fecal microbiota preparation that satisfied all our preset objectives, including preservation of viability across all bacterial phyla, satisfactory physicochemical characteristics (a finely divided powder) that enabled easy encapsulation, and excellent clinical efficacy. We formally demonstrated that the preparation remains stable for 4 days at a range of temperatures, which is time sufficient to allow flexibility in dispensing to patients, and there is every expectation that it will remain stable for much longer periods as long as the environment is dry. We kept the capsules in a $-80{ }^{\circ} \mathrm{C}$ laboratory freezer merely for convenience since it does provide a very dry environment. Additional more long-term storage experiments are ongoing.

The clinical experience was pragmatic, although we excluded patients with known inflammatory bowel disease. We made a number of adjustments in the FMT capsule treatment protocol since its original introduction into our clinical program, including elimination of the colon purgative, allowing patients to stay on their proton pump inhibitor, and lowering the dose of microbiota by an order of magnitude. None of these changes have made a detectable impact on the clinical efficacy or kinetics of fecal microbiota changes following FMT. The resulting protocol, which has now become standard in our program, is remarkably simple, necessitating a single ingestion of several capsules containing fecal microbiota on an empty stomach 2 days after discontinuation of vancomycin.

The clinical experience in this study was not constructed as a formal, explanatory clinical trial. Nevertheless, the patients had very close follow-up including systematic home visits by the research assistant for a clinical check-up and collection of fecal samples. All patients were also seen in clinic 2 months after capsule FMT and remain in contact with the clinic with any questions or concerns. The study design was adequate to capture serious adverse events. As noted, one patient with urinary stents developed a urinary tract infection necessitating hospitalization. It is unlikely that FMT contributed to this problem. The rate of minor gastrointestinal symptoms such as irregular bowel movements, bloating, and flatulence during the initial weeks following capsule administration was comparable to observations made in other post-FMT experiences and formal clinical trials $(6,7,10,33,36)$, and likely represented some element of post-infectious IBS (37). Long-term follow-up studies are needed to determine the natural history of post-infectious IBS in this patient population.

The capsule FMT resulted in normalization of the fecal microbial community structure. However, it is interesting that the kinetics of microbiome changes was delayed, especially for Bacteroidetes, compared to colonoscopic FMT where full donor microbiota engraftment is evident within 1 day (25). This was surprising since recipient germ-free mice did not demonstrate any differences among the various bacterial phyla in kinetics of engraftment. 
Nevertheless, the clinical success of capsule FMT suggests that prompt recovery of Bacteroidetes is not required to prevent CDI recurrence.

The small failure rate for capsule FMT is comparable to our colonoscopic experience. The reasons for these failures remain elusive. Thus far we did not identify a pattern of microbiota composition following FMT that would be predictive of FMT failure. Given the high rate of home contamination of R-CDI patients with $C$. difficile spores (38), it is possible that patients remain at risk for re-infection during the initial weeks after FMT before establishment of the new microbial community and its functionality. We are also currently investigating the possibility that the specific $C$. difficile strains from these patients are less responsive to secondary bile-mediated inhibition of germination and growth.

Our study does have some important limitations. This was a small, single center, pragmatic study to collect initial clinical experience with capsule FMT, not a trial to determine clinical efficacy of capsules vs. colonoscopic FMT or another therapeutic preparation. Based on the efficacy data observed here, a randomized, controlled, double-blind clinical trial to determine effectiveness (or non-inferiority) of capsules vs. other forms of FMT would require a very large study. The protocol changed over time based on our early experiences and intrinsic constraints. While we noted no difference in our cohort between the low dose and high dose capsule FMT, it is possible that larger studies could identify a more subtle difference. A similar statement applies to concurrent use of proton pump inhibitors.

In summary, easy-to-handle capsules containing freeze-dried fecal microbiota provide a safe and highly clinically effective treatment of refractory R-CDI. This form of FMT results in somewhat delayed normalization of recipient fecal microbial community structure compared to colonoscopic FMT. However, this difference does not appear to impair clinical response with respect to prevention of CDI recurrence. Clearly, next-generation FMT preparations will continue to be developed, and detailed analysis of microbiota engraftment and functionality will continue to be useful in guiding this work. Although the patient population was somewhat heterogeneous and the numbers of patients in different subgroups was small, the experience provides a foundation for the design of large, controlled, explanatory clinical trials. The FMT preparation described here is certainly easily adaptable to further rigorous study.

\section{Supplementary Material}

Refer to Web version on PubMed Central for supplementary material.

\section{Acknowledgments}

This research was made possible by support by grants from the NIH 1R21-AI114722-01 (AK, MJS), CIPAC Limited (AK, MJS), Achieving Cures Together, Ms. Emily Haller (AK), and the Hubbard Foundation (AK).

\section{References}

1. Spellberg B, Guidos R, Gilbert D, et al. The epidemic of antibiotic-resistant infections: a call to action for the medical community from the Infectious Diseases Society of America. Clin Infect Dis. 2008; 46:155-64. [PubMed: 18171244] 
2. Fauci AS, Marston LD. The perpetual challenge of antimicrobial resistance. JAMA. 2014; 311:1853-4. [PubMed: 24652442]

3. Pamer EG. Resurrecting the intestinal microbiota to combat antibiotic-resistant pathogens. Science. 2016; 352:535-8. [PubMed: 27126035]

4. Borody TJ, Khoruts A. Fecal microbiota transplantation and emerging applications. Nat Rev Gastroenterol Hepatol. 2012; 9:88-96.

5. Drekonja D, Reich J, Gezahegn S, et al. Fecal microbiota transplantation for Clostridium difficile infection: a systematic review. Ann Intern Med. 2015; 162:630-8. [PubMed: 25938992]

6. van Nood E, Vrieze A, Nieuwdorp M, et al. Duodenal infusion of donor feces for recurrent Clostridium difficile. N Engl J Med. 2013; 368:407-15. [PubMed: 23323867]

7. Hamilton MJ, Weingarden AR, Sadowsky MJ, et al. Standardized frozen preparation for transplantation of fecal microbiota for recurrent Clostridium difficile infection. Am J Gastroenterol. 2012; 107:761-7. [PubMed: 22290405]

8. Khoruts A, Rank KM, Newman KM, et al. Inflammatory bowel disease affects the outcome of fecal microbiota transplantation for recurrent Clostridium difficile infection. Clin Gastroenterol Hepatol. 2016; 14:1433-8. [PubMed: 26905904]

9. Zipursky JS, Sidorsky TI, Freedman CA, et al. Patient attitudes toward the use of fecal microbiota transplantation in the treatment of recurrent Clostridium difficile infection. Clin Infect Dis. 2012; 55:1652-8. [PubMed: 22990849]

10. Youngster I, Russell GH, Pindar C, et al. Oral, capsulized, frozen fecal microbiota transplantation for relapsing Clostridium difficile infection. JAMA. 2014; 312:1772-8. [PubMed: 25322359]

11. Sogin ML, Morrison HG, Huber JA, et al. Microbial diversity in the deep sea and the underexplored "rare biosphere". Proc Natl Acad Sci USA. 2006; 103:12115-20. [PubMed: 16880384]

12. Claesson MJ, Wang Q, O’Sullivan O, et al. Comparison of two next-generation sequencing technologies for resolving highly complex microbiota composition using tandem variable $16 \mathrm{~S}$ rRNA gene regions. Nucleic Acids Res. 2010; 38:e200. [PubMed: 20880993]

13. Schloss PD, Westcott SL, Ryabin T, et al. Introducing mothur: open-source, platform-independent, community-supported soft ware for describing and comparing microbial communities. Appl Environ Microbiol. 2009; 75:7537-41. [PubMed: 19801464]

14. Staley C, Gould TJ, Wang P, et al. Evaluation of water sampling methodologies for amplicon-based characterization of bacterial community structure. J Microbiol Methods. 2015; 114:43-50. [PubMed: 25956022]

15. Aronesty E. Comparison of sequencing utility programs. Open Bioinforma J. 2013; 7:1-8.

16. Pruesse E, Quast C, Knittel K, et al. SILVA: a comprehensive online resource for quality checked and aligned ribosomal RNA sequence data compatible with ARB. Nucleic Acids Res. 2007; 35:7188-96. [PubMed: 17947321]

17. Huse SM, Welch DM, Morrison HG, et al. Ironing out the wrinkles in the rare biosphere through improved OTU clustering. Environ Microbiol. 2010; 12:1889-98. [PubMed: 20236171]

18. Edgar RC, Haas BJ, Clemente JC, et al. UCHIME improves sensitivity and speed of chimera detection. Bioinformatics. 2011; 27:2194-200. [PubMed: 21700674]

19. Gihring TM, Green SJ, Schadt CW. Massively parallel rRNA gene sequencing exacerbates the potential for biased community diversity comparisons due to variable library sizes. Environ Microbiol. 2012; 14:285-90. [PubMed: 21923700]

20. Cole JR, Wang Q, Cardenas E, et al. The Ribosomal Database Project: improved alignments and new tools for rRNA analysis. Nucleic Acids Res. 2009; 37:D141-5. [PubMed: 19004872]

21. Knights D, Kuczynski J, Charlson ES, et al. Bayesian community-wide culture-independent microbial source tracking. Nat Methods. 2011; 8:761-3. [PubMed: 21765408]

22. Excoffier L, Smouse PE, Quattro JM. Analysis of molecular variance inferred from metric distances among DNA haplotypes: application to human mitochondrial DNA restriction data. Genetics. 1992; 131:479-91. [PubMed: 1644282]

23. Clarke KR. Non-parametric multivariate analyses of changes in community structure. Aust J Ecol. 1993; 18:117-43. 
24. Hubalek Z. Protectants used in the cryopreservation of microorganisms. Cryobiology. 2003; 46:205-29. [PubMed: 12818211]

25. Weingarden A, Gonzalez A, Vazquez-Baeza Y, et al. Dynamic changes in short- and long-term bacterial composition following fecal microbiota transplantation for recurrent Clostridium difficile infection. Microbiome. 2015; 3:10. [PubMed: 25825673]

26. Hamilton MJ, Weingarden AR, Unno T, et al. High-throughput DNA sequence analysis reveals stable engraftment of gut microbiota following transplantation of previously frozen fecal bacteria. Gut Microbes. 2013; 4:125-35. [PubMed: 23333862]

27. Weingarden AR, Chen C, Bobr A, et al. Microbiota transplantation restores normal fecal bile acid composition in recurrent Clostridium difficile infection. Am J Physiol Gastrointest Liver Physiol. 2014; 306:G310-9. [PubMed: 24284963]

28. Shankar V, Hamilton MJ, Khoruts A, et al. Species and genus level resolution analysis of gut microbiota in Clostridium difficile patients following fecal microbiota transplantation. Microbiome. 2014; 2:13. [PubMed: 24855561]

29. Shahinas D, Silverman M, Sittler T, et al. Toward an understanding of changes in diversity associated with fecal microbiome transplantation based on 16S rRNA gene deep sequencing. MBio. 2012 th:e00338-12.

30. Seekatz AM, Aas J, Gessert CE, et al. Recovery of the gut microbiome following fecal microbiota transplantation. MBio. 2014; 5:e00893-14. [PubMed: 24939885]

31. Surawicz CM, Brandt LJ, Binion DG, et al. Guidelines for diagnosis, treatment, and prevention of Clostridium difficile infections. Am J Gastroenterol. 2013; 108:478-98. quiz 499. [PubMed: 23439232]

32. Lee CH, Steiner T, Petrof EO, et al. Frozen vs. fresh fecal microbiota transplantation and clinical resolution of diarrhea in patients with recurrent Clostridium difficile infection: a randomized clinical trial. JAMA. 2016; 315:142-9. [PubMed: 26757463]

33. Youngster I, Sauk J, Pindar C, et al. Fecal microbiota transplant for relapsing Clostridium difficile infection using a frozen inoculum from unrelated donors: a randomized, open-label, controlled pilot study. Clin Infect Dis. 2014; 58:1515-22. [PubMed: 24762631]

34. Youngster I, Mahabamunuge J, Systrom HK, et al. Oral, frozen fecal micro-biota transplant (FMT) capsules for recurrent Clostridium difficile infection. BMC Med. 2016; 14:134. [PubMed: 27609178]

35. Hirsch BE, Saraiya N, Poeth K, et al. Effectiveness of fecal-derived micro-biota transfer using orally administered capsules for recurrent Clostridium difficile infection. BMC Infect Dis. 2015; 15:191. [PubMed: 25885020]

36. Orenstein R, Dubberke E, Hardi R, et al. Safety and durability of RBX2660 (microbiota suspension) for recurrent Clostridium difficile infection: results of the PUNCH CD study. Clin Infect Dis. 2016; 62:596-602. [PubMed: 26565008]

37. Wadhwa A, Al Nahhas MF, Dierkhising RA, et al. High risk of post-infectious irritable bowel syndrome in patients with Clostridium difficile infection. Aliment Pharmacolfier. 2016; 44:57682.

38. Shaughnessy MK, Bobr A, Kuskowski MA, et al. Environmental contamination in households of patients with recurrent Clostridium difficile. Infection. 
Study Highlights

\section{WHAT IS CURRENT KNOWLEDGE}

- $\quad$ Fecal microbiota transplantation (FMT) can be accomplished by administration of a frozen preparation by different routes.

- Little is known about dosing of FMT or engraftment of different preparations.

\section{WHAT IS NEW HERE}

- $\quad$ An optimized, stable freeze-dried preparation of fecal microbiota is comparable in clinical efficacy to other FMT preparations in treatment of recurrent Clostridium difficile infection in this observational study.

- $\quad$ Low dose (2-4 capsules) preparation administered as a single dose without colon purgative is as efficacious as high dose (24-27 capsules) in clinical efficacy and engraftment. 


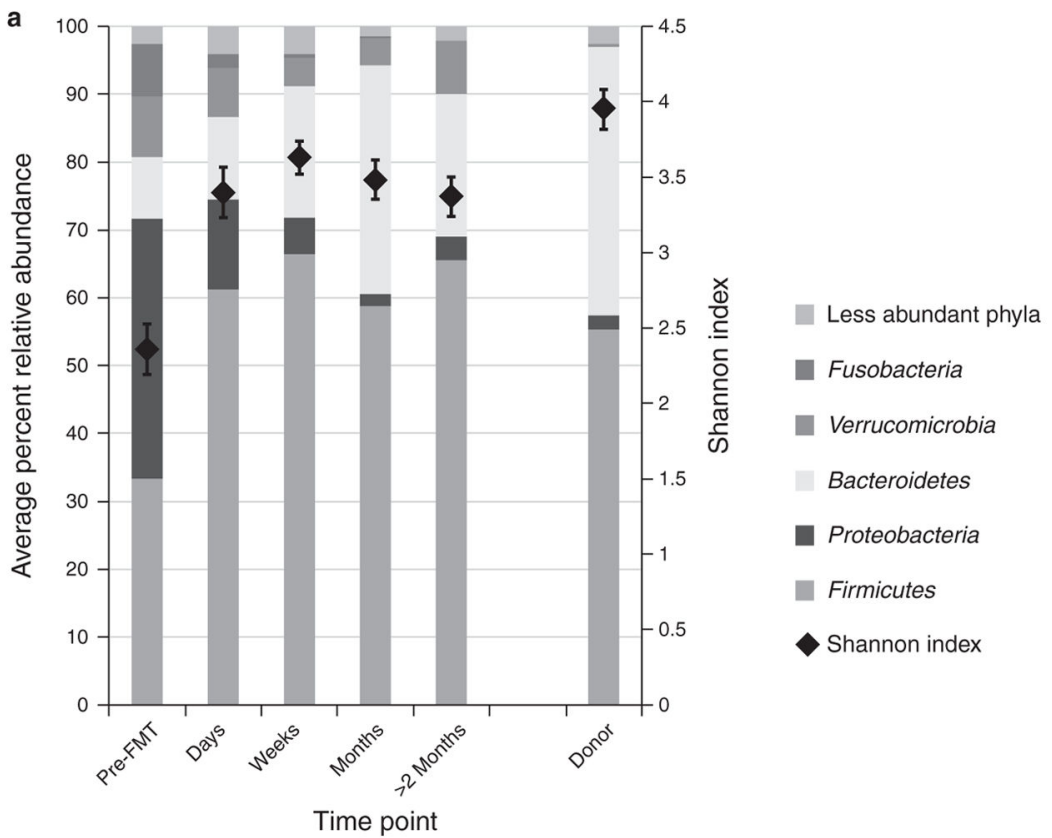

b

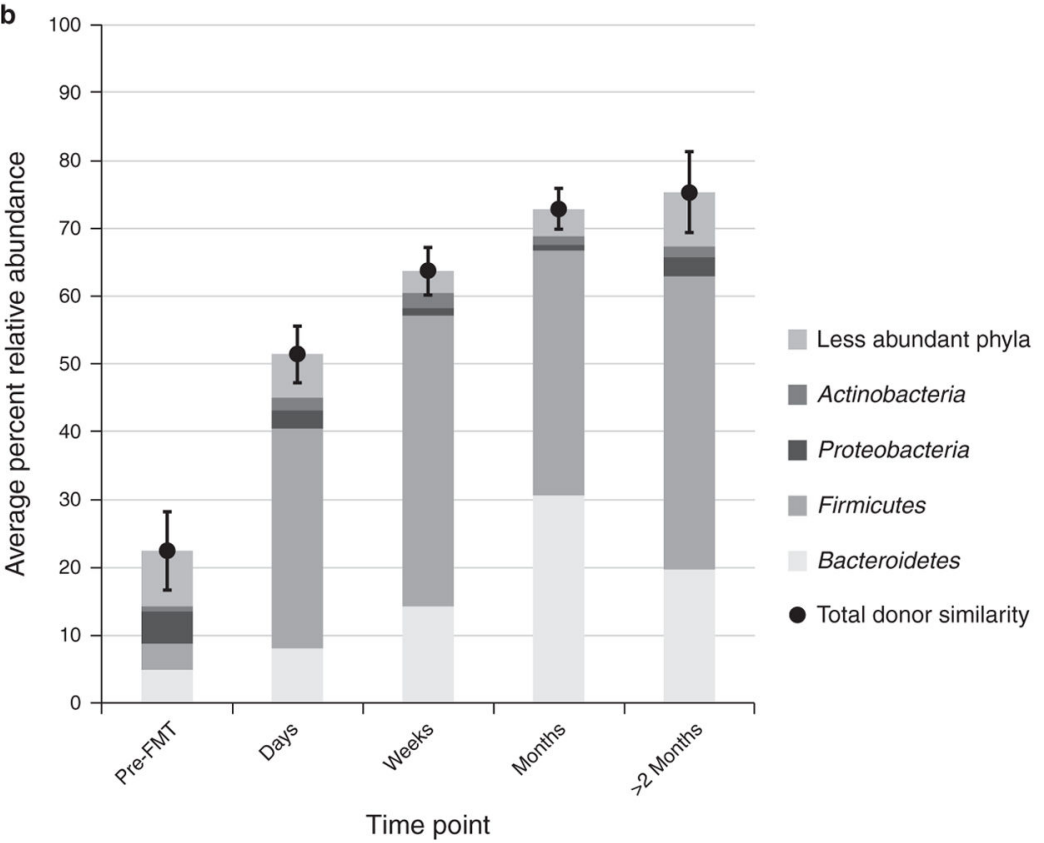

Figure 1.

Changes in the fecal bacterial composition associated with capsule FMT. (a) Distribution of phyla in fecal samples from successfully treated patients and donors. Samples were collected before fecal microbial transplantation (pre-FMT), within the first 6 days post-FMT (days), between 7 and 21 days post-FMT (weeks), between 30 and 60 days post-FMT (months), or after 2 months post-FMT ( $>2$ months). (b) Phylum-level classification of operational taxonomic units (OTUs) that were associated with donor contribution as estimated by SourceTracker. Error bars reflect s.e.m. 
Table 1

Clinical characteristics of patients treated with capsule FMT

\begin{tabular}{|c|c|c|}
\hline & All patients, $n=49$ & First FMT via capsule, $n=39$ \\
\hline \multicolumn{3}{|l|}{ Age (years) } \\
\hline Mean \pm s.d. & $62.3 \pm 17.1$ & $63.8 \pm 18.1$ \\
\hline \multicolumn{3}{|l|}{$\operatorname{Sex}, n(\%)$} \\
\hline Female & $43(87.8 \%)$ & $34(87.2 \%)$ \\
\hline \multicolumn{3}{|l|}{ Time from initial CDI diagnosis (months) } \\
\hline Median (range) & $10.0(3-54)$ & $10.0(3-54)$ \\
\hline History of hospitalization for CDI, $n(\%)$ & $11(22.4 \%)$ & $9(23.1 \%)$ \\
\hline \multicolumn{3}{|l|}{ Antibiotics used to treat $C D I$} \\
\hline Metronidazole & $75.5 \%$ & $82.1 \%$ \\
\hline Vancomycin & $100 \%$ & $100 \%$ \\
\hline Fidaxomicin & $26.5 \%$ & $17.9 \%$ \\
\hline Rifaximin & $6.1 \%$ & $5.1 \%$ \\
\hline Intercurrent non-CDI antibiotics ${ }^{a}$ & $28.5 \%$ & $30.8 \%$ \\
\hline Probiotics & $49.0 \%$ & $56.4 \%$ \\
\hline \multicolumn{3}{|l|}{ Additional medications } \\
\hline Proton pump inhibitors & $18.4 \%$ & $20.5 \%$ \\
\hline HMG-CoA reductase inhibitors & $26.5 \%$ & $23.1 \%$ \\
\hline
\end{tabular}

CDI, Clostridium difficile infection; CoA, coordinate analysis; FMT, fecal microbial transplantation.

${ }^{a}$ Intercurrent non-CDI antibiotics were antibiotics given for a non-CDI indication during the period following the initial CDI and FMT. However, recurrence of CDI associated with a non-CDI antibiotic was not considered to be a spontaneous CDI recurrence and did not qualify patients for FMT. 


\section{Table 2}

Clinical protocol variables in administering capsule FMT

\begin{tabular}{|lc|}
\hline Clinical variable & Success rate $^{\boldsymbol{a}}$ (percent) \\
\hline Purgative before FMT & $3 / 4(75.0 \%)$ \\
\hline Yes & $40 / 45(88.9 \%)$ \\
\hline No & \\
\hline History of past colonoscopic FMT & $8 / 10(80.0 \%)$ \\
\hline Yes & $35 / 39(89.7 \%)$ \\
\hline No & \\
\hline Proton pump inhibitor & $9 / 10(90.0 \%)$ \\
\hline Yes & $35 / 39(89.7 \%)$ \\
\hline No & $15 / 19(78.9 \%)$ \\
\hline High dose $\left(1.25-2.5 \times 10^{12}\right.$ bacteria) & $28 / 30(93.3 \%)$ \\
\hline Low dose $\left(2.1-2.5 \times 10^{11}\right.$ bacteria) & \\
\hline
\end{tabular}

CDI, Clostridium difficile infection; FMT, fecal microbial transplantation.

${ }^{a}$ Success of FMT was defined as no recurrence of CDI as described in the Methods section within 2 months of follow-up following treatment. None of the differences reached statistical significance. 